\title{
Os Benefícios da Aplicação de Jogos Educacionais Executados por Computador e Dispositivos Móveis no Tratamento do Transtorno do Espectro Autista
}

\author{
Rhaylson Silva do Nascimento ${ }^{1}$ \\ ${ }^{1}$ Instituto Federal de Educação, Ciência e Tecnologia do Piauí (IFPI) \\ CEP 64860-000 - Uruçuí - PI - Brasil \\ rhaylson.silvadifpi.edu.br
}

\begin{abstract}
In Brazil, almost 500 thousand people under 20 years old suffer with autism. This chronic disorder is characterized by symptoms like language disorders, difficulties to communicate, repetitive behaviors, amongst others. Educational ludo games, executed in computer or mobile devices, are presented as alternatives to improve the quality of life of patients with ASD, teaching them new skills that will assist them in the execution of their daily activities. In this work, the importance of this type of approach is discussed, highlighting its contribution to those with ASD and team members participating in the treatment.
\end{abstract}

Resumo. No Brasil, estima-se que em torno de 500 mil pessoas abaixo dos 20 anos sofram de autismo. Esse transtorno crônico caracteriza-se por alguns sintomas que vão desde perturbações na linguagem, dificuldade de comunicação, comportamentos repetitivos, entre outros. Jogos ludo educativos, executados por computador ou dispositivos móveis, apresentam-se como alternativas para melhorar a qualidade de vida dos portadores, ensinando-os novas habilidades que os auxiliarão na execução de suas atividades cotidianas. Nesse trabalho, discute-se a importância desse tipo de abordagem, destacando sua contribuição para os portadores do TEA e a membros da equipe que participam do tratamento.

\section{Introdução}

Segundo dados da organização das nações unidas (ONU), existem atualmente 70 milhões de pessoas no mundo acometida pelo autismo [Carvalho 2018]. No Brasil, estima-se que são em torno de 500 mil indivíduos abaixo dos 20 anos, sendo 115 mil crianças de 0 a 4 anos [Cunha 2011]. Esse transtorno crônico, cuja origem ainda vem sendo discutida por cientistas, caracteriza-se por alguns sintomas que variam desde perturbações na linguagem, dificuldade de comunicação, comportamentos repetitivos e uma necessidade de manter o ambiente ao qual se encontram imutável.

Estudantes acometidos por TEA, crianças principalmente, apresentam uma série de deficiências qualitativas de comunicação e interação social que dificultam sua inserção em um ambiente escolar comum [Silva et al. 2017]. Segundo Silva et al. (2017), tais alunos podem apresentar oposição quanto a interação social, além de 
dificuldade na compreensão dos significados das palavras e também na identificação de emoções.

As novas tecnologias da informação e comunicação representam uma possibilidade de inovação para auxílio de pessoas que carecem de medidas educativas especiais [Santana 2016]. Computadores e dispositivos móveis vêm sendo utilizadas por profissionais da saúde visando a aplicação de tratamentos direcionados aos portadores do autismo. O objetivo nesse tipo de abordagem é a melhora das habilidades cognitivas dos pacientes afetados pelo transtorno e a aquisição de novos conhecimentos básicos, como a leitura, escrita, reconhecimento de padrões, cores e emoções. O presente artigo tem como objetivo discutir a importância desse tipo de tratamento na vida de portadores do TEA, destacando suas contribuições na aquisição de habilidades simples que fazem parte da rotina do autista na sociedade. Além disso, mostrar as contribuições que essa intervenção fornece aos profissionais que fazem parte da equipe que realiza o tratamento.

\section{Desenvolvimento}

Pessoas afetadas por TEA demonstram uma variação quanto ao nível de desenvolvimento intelectual. De acordo com Mello e Sganzerla. (2013), percebe-se em autistas o comprometimento das atividades escolares, pois costumam demonstrar uma aprendizagem mais centrada em poucos assuntos com predominância do pensamento concreto, havendo também o comprometimento da comunicação e da interação social. A tecnologia da informação quando aplicada ao autismo se mostra bastante promissora, pois ajuda os profissionais a acompanhar o tratamento e também a desenvolver atividades na forma de jogos e atividades ludo educativas, executadas por computador e dispositivos móveis, capazes de melhorar o comportamento e as capacidades cognitivas dos portadores do transtorno.

Segundo Cunha (2011), esse tipo de intervenção quando realizada até os três anos de idade, diminui os déficits do autista, isto é, comportamentos anormais associados ao transtorno, permitindo ao portador maior independência e qualidade de vida. Entretanto, não é qualquer atividade executada em um ambiente tecnológico que se mostra aproveitável para o ensino de novas habilidades a portadores de TEA. Toda e qualquer intervenção deverá sempre pautar-se em estudos anteriores e em métodos que se mostraram eficientes em ambiente clínico e educacional. Conhecimentos da informática tais como linguagem de programação, fundamento da gamificação e tecnologias assistivas precisam estar interligados as demais áreas do conhecimento para que sua aplicação seja inteiramente eficaz durante o tratamento. Tal fato é algo previsível do ponto de vista lógico, visto que o tratamento de um paciente acometido por TEA envolve equipes multidisciplinares compostas por profissionais diversos como pedagogos, psicólogos, fonoaudiólogos, fisioterapeutas, médicos, dentre outras profissões da saúde e educação como frisa Carvalho (2011).

A seguinte dúvida pode aparecer: seria mesmo necessário aplicar esse novo método de tratamento em autistas mesmo com os aqueles já tradicionais demonstrandose eficazes? A resposta seria sim, pois a tecnologia da informação não surge como elemento substituto do tratamento tradicional, mas sim como item auxiliar. Como destaca Cunha (2011), o uso de computadores e dispositivos móveis permite a criação 
de ambientes controlados, interessantes e sem distrações para execução de atividades. Esse tipo de ambiente favorece o aprendizado, visto que chama a atenção do indivíduo e mantém seu foco em uma atividade específica. Algumas vezes, pode haver repulsa do paciente, como demonstrado por Neto et al. (2013), quando crianças que não estavam familiarizadas com atividades executadas em um tablet rejeitaram o uso de uma aplicação para o ensino de cores, mas, no geral, a grande maioria dos pacientes se mostra interessada e motivada em realizar esse tipo de atividade [Cunha 2013] [Silva et al. 2017]. Para Silva et al. (2016), esse interesse se dá por conta de características presentes nos dispositivos móveis e computadores. Suas cores, luzes e telas com função de toque se mostram como algo novo e chamativo e motivador. Sabendo utilizar tais características em conjunto com conhecimento de outras áreas da saúde e educação se torna possível criar soluções interessantes para um tipo de tratamento mais direcionado. Contudo, como mencionado antes, toda e qualquer atividade planejada no ambiente tecnológico precisa estar pautada em teorias e tratamentos tradicionais pré-existentes.

Hoje, a maioria das abordagens computacionais descrevem soluções baseadas no método conhecido como ABA (Applied Behavior Analysis), em português Análise de Comportamento Aplicada, uma técnica bastante intuitiva da psicologia que visa a aquisição e desenvolvimento de atividades cognitivas, comunicacionais e sociais através de repetições controladas de um mesmo exercício [Silva et al. 2016]. O ABA consiste em dividir uma atividade em partes menores, treinar essa habilidade repetidas vezes e no fim recorrer a reforços para inibição de comportamentos indesejados [Neto et al. 2013].

$\mathrm{Na}$ sua forma tradicional, a aplicação do ABA é feita através de atividades mecânicas, com uso de papel, brinquedos, jogos de tabuleiro. Contudo, as novas tecnologias da informação vêm modificando essa forma tradicional de aplicação. Percebe-se na literatura o esforço na criação de jogos educativos baseados no método por grupos da área da tecnologia da informação. Soluções descritas em trabalhos publicados por Cunha (2011), Neto et al. (2013), Silva et al.(2016), como o ensino de cores baseado em repetições controladas, o aprendizado de atividades pré-acadêmicas através da formação de palavras, da leitura e identificação de objetos através de imagens, e aplicações que visam o desenvolvimento da fala servem para que pacientes autistas jovens aprendam a realizar atividades do dia a dia, de forma independente, aumentando suas capacidades.

É possível que algumas pessoas, sem nenhum tipo de deficiência cognitiva, afirmem que esses tipos de atividades são coisas triviais, visto sua simplicidade, mas para o autistas são de extrema importância para melhora da qualidade de vida. Os profissionais da área da saúde também recebem com entusiasmo essas novas aplicações. Como apresenta Silva e Soares e Benitez (2017), psicólogos percebem que seus pacientes apresentaram mais interesse em usar recursos tecnológicos nas suas atividades e que o desenvolvimento das habilidades treinadas durante o tratamento só vêm a melhorar à medida que o autista se familiariza com essas novas ferramentas.

Outro destaque se refere à facilidade em monitorar a evolução de pacientes treinados, devido, principalmente, à natureza dos sistemas computacionais. Quando se executa algo em um ambiente controlado e informacional todo e qualquer dado pode ser armazenado, logo, tudo aquilo que foi testado durante as atividades desenvolvidas pode ser analisado posteriormente de forma simples e dinâmica. A criação de relatórios de 
acompanhamento, de gráficos e de outros artefatos que ajudam equipes multidisciplinares que realizam o tratamento de autistas pode ser automatizada com o uso de computadores e dispositivos móveis como demonstrado por Neto et al. (2013) e Silva et al. (2016). Isso facilita a tomada de decisão e ajuda a direcionar o tratamento. A utilização de atividades assistidas por computadores e dispositivos móveis se mostram muito eficientes tanto para o paciente quanto os profissionais envolvidos no tratamento do TEA, trazendo qualidade de vida ao portador e comodidade aos profissionais. Desse modo, a existência desses novos métodos se torna totalmente necessária.

\section{Conclusão}

Diante do que foi levantado fica claro que a informática surge como uma grande aliada para o tratamento e auxílio de portadores do transtorno de espectro autista. As técnicas da computação, quando aplicadas corretamente, ajudam profissionais a propor tratamentos mais direcionados aos pacientes acometidos pelo transtorno e a monitorar sua evolução. Utilizando computadores e dispositivos móveis é possível trazer mais qualidade de vida aos pacientes portadores do TEA. Como trabalho futuro, pretende-se revisar outras soluções voltadas para a aquisição de habilidades motoras e sociais baseadas na robótica e realidade aumentada.

\section{Agradecimentos}

Ao Instituto Federal do Piaú por financiar a pesquisa através de edital de fomento PIBEX 065/2015 e ao Grupo de pesquisas em ciências humanas e linguagens do cerrado.

\section{Referências}

Carvalho, L. R. (2018) "SATA- sistema de acompanhamento de tratamento de autismo", 2018. Monografia (Graduação em Tecnologia em Análise e Desenvolvimento de Sistemas) Instituto Federal de Educação, Ciência e Tecnologia do Piauí, Teresina - Piauí.

Cunha, R.M. (2011). "Desenvolvimento e avaliação de um jogo de computador para ensino de vocabulário para crianças com autismo", Proceedings of Games for Change.

Silva, M e SOARES, A e BENITEZ, P (2017). "Ambiente Digital para Ensino e Acompanhamento Personalizado de Estudantes com Autismo: proposta com Uso de Dispositivos Móveis". In: Brazilian Symposium on Computers in Education (Simpósio Brasileiro de Informática na Educação-SBIE). p. 1047.

Silva, M. et al. (2016). "Ambiente digital para ensino de atividades (pré)acadêmicas para estudantes com autismo". In: ANAIS DO $7^{\circ}$ CONGRESSO BRASILEIRO DE EDUCAÇÃO ESPECIAL, Campinas, GALOÁ.

Mello, C. M. C. e Sganzerla, M. A. R. (2013). "Aplicativo Android para auxiliar no desenvolvimento da comunicação de Autistas". In: XVIII Congresso Internacional de Informática Educativa. p. 231-239.

Neto, S. P. O. et al. (2013). "G-TEA: Uma ferramenta no auxílio da aprendizagem de crianças com Transtorno do Espectro Autista, baseada na metodologia ABA". SBCProceedingsofSBGames, 2013.

Santana, F. C. B et al.(2016). "Diagnóstico para Tratamento do Transtorno do Espectro Autista para com base em Lógica Nebulosa". In: CONGRESSO NORTE NORDESTE DE PESQUISA E INOVAÇÃO, 11. Maceió. Anais... Maceió: IFAL. p. 918-924. 